\title{
A model of chronic inflammation in atherosclerosis
}

\author{
Vladimir Bezyaev ${ }^{1, *}$, Nail Sadekov ${ }^{1, * *}$, and Vitaly Volpert ${ }^{1,2, * * *}$ \\ ${ }^{1}$ RUDN University, 6 Miklukho-Maklaya St, Moscow, 117198, Russian Federation \\ ${ }^{2}$ Institut Camille Jordan, UMR 5208 CNRS, University Lyon 1, Villeurbanne 69622, France
}

\begin{abstract}
Atherosclerosis is a chronic progressive disease leading to the formation of atherosclerotic plaques in artery walls, narrowing its lumen. In this work, we present a mathematical model of atherosclerosis development for the concentrations of cells and cytokines of innate immunity. This system describes a chronic inflammatory response in the intima of an artery vessel wall. The stationary points and their stability for reduced kinetic systems of two and five ODEs are investigated. The relationship of the obtained results is analyzed, and their biological interpretation is given.
\end{abstract}

\section{Introduction}

Cardiovascular diseases related to atherosclerosis represent one of the major causes of morbidity and mortality worldwide. Although some progress has been made in diagnosis, surgical treatment and control of high cholesterol level, a decisive progress to fight atherosclerosis has not yet been achieved. Coronary heart disease and stroke result from a sharp decrease in blood supply due to atherosclerotic plaque or related thrombosis and vessel occlusion [1].

The process of plaque development can begin with the damage of the endothelial layer, allowing low density lipoproteins (LDL) to pass from the blood into the intima with further oxidizing by free radicals $[2,3]$. Sensing the presence of the oxidized LDL (ox-LDL) in the intima, endothelial cells secrete monocyte chemoattractant protein (MCP-1), which promotes the recruitment of monocytes in the intima [4]. After entering the intima, monocytes differentiate into macrophages, which endocytose the ox-LDL [5-7]. A large amount of ox-LDL in the vessel wall promotes the transformation of macrophages into foam cells $[2,3]$. Macrophages $M_{1}$ produce pro-inflammatory cytokines such as IL-6, IL-12 and TNF- $\alpha$ and the alternatively activated macrophages $M_{2}$ produce anti-inflammatory cytokines such as IL-10 and TGF- $\beta$ [6]. Pro-inflammatory cytokines contribute to plaque growth activating $T_{1}$-helper lymphocytes [8] that produce pro-inflammatory cytokines such as IFN- $\gamma$ and IL- $1 \beta$ and stimulating $M_{1}$ macrophages differentiation, whereas $T_{2}$-helper cells produce cytokines such as IL-4 and IL-13 required for $M_{2}$ macrophages differentiation [6]. Together with LDL, high density lipoproteins (HDL) also enter the intima. HDL acts to prevent the development of atherosclerosis taking free radicals from oxidizing LDL and possibly removing cholesterol from foam cells [9].

\footnotetext{
*e-mail: vbezyaev@mail.ru

**e-mail: nail.sadd@mail.ru

***e-mail: volpert@math.univ-lyon1.fr
} 


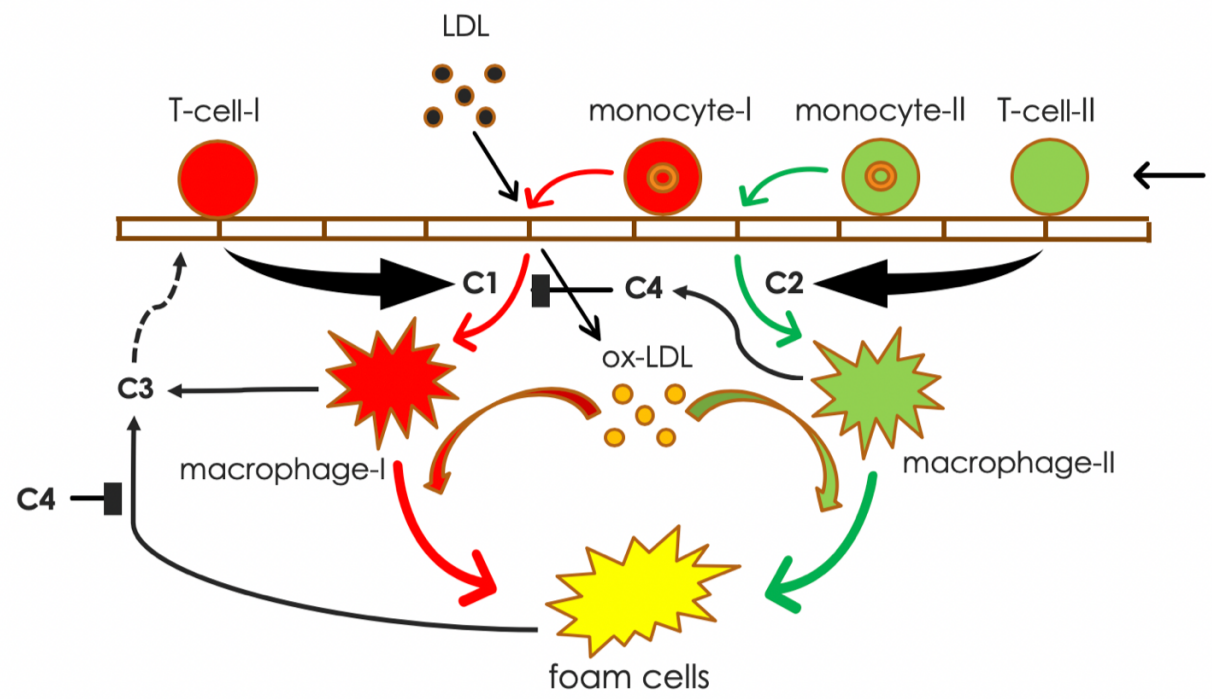

Figure 1. Schematic network of atherosclerosis. LDL in the intima are oxidized and converted to ox-LDL. Ox-LDL activate endothelial cells leading to the recruitment of monocytes. By ingesting ox$\mathrm{LDL}$, macrophages are transformed into foam cells. Cytokines $C_{1}$ and $C_{2}$ secreted by T-cells enhance the activity of macrophages. Macrophages $M_{1}$ and foam cells $F$ secrete pro-inflammatory agents $C_{3}$, which activates T-cells. Macrophages $M_{2}$ secrete anti-inflammatory agents $C_{4}$, which inhibit T-cells activation.

\section{Mathematical model}

In this work, we present a mathematical model of atherosclerosis development based on a ODE system for the concentrations of cells and cytokines of innate immunity. This system describes a chronic inflammatory reaction in the intima of the artery wall. The mathematical model is based on the processes schematically shown in Figure 1. The model includes the variables listed in Table 1 and the following equations:

Equation for LDL (low density lipoproteins):

$$
\frac{d L}{d t}=\sigma_{L}\left(L_{0}-L\right)-k_{L} r L
$$

Here $\sigma_{L}$ is rate of LDL penetration through the vessel wall, $L_{0}$ is the concentration of LDL in the blood, $k_{L}$ is the reaction rate of oxidization, where LDL is consumed [2,3].

Equation for HDL (high density lipoproteins):

$$
\frac{d H}{d t}=\sigma_{H}\left(H_{0}-H\right)-k_{H} r H
$$

Here $\sigma_{H}$ is rate of HDL penetration through the vessel wall, $H_{0}$ is the concentration of HDL in the blood, $k_{H}$ is the reaction rate of oxidization. HDL comes from the blood and it is consumed in the oxidation reaction with free radicals $[2,3]$.

Equation for ox-LDL (oxidized low density lipoproteins):

$$
\frac{d L_{o x}}{d t}=k_{L} r L-\lambda_{L_{o x} M_{1}} \frac{L_{o x}}{k_{L_{o x}}+L_{o x}} M_{1}-\lambda_{L_{o x} M_{2}} \frac{L_{o x}}{k_{L_{o x}}+L_{o x}} M_{2}-d_{L_{o x}} L_{o x} .
$$


Table 1. The variables of the model

\begin{tabular}{ll}
\hline Variable & Description $\left[\mathrm{g} / \mathrm{cm}^{3}\right]$ \\
\hline$L$ & Concentration of LDL \\
$H$ & Concentration of HDL \\
$L_{o x}$ & Concentration of ox-LDL \\
$r$ & Concentration of free radicals \\
$A_{1}$ & Density of $A_{1}$ monocytes \\
$A_{2}$ & Density of $A_{2}$ monocytes \\
$M_{1}$ & Density of $M_{1}$ macrophages \\
$M_{2}$ & Density of $M_{2}$ macrophages \\
$T_{1}$ & Density of $T_{1}$-helper cells \\
$T_{2}$ & Density of $T_{2}$-helper cells \\
$C_{1}$ & Concentration of $T_{1}$-helper cytokines IFN- $\gamma$, IL-1 $\beta$ \\
$C_{2}$ & Concentration of $T_{2}$-helper cytokines IL-4, IL-13 \\
$C_{3}$ & Concentration of pro-inflammatory agents IL-6, IL-12 and TNF- $\alpha$ \\
$C_{4}$ & Concentration of anti-inflammatory agents IL-10 and TGF- $\beta$ \\
$F$ & Density of foam cells \\
\hline
\end{tabular}

Here $\lambda_{L_{o x} M_{1}}$ and $\lambda_{L_{o x} M_{2}}$ are the rates of ox-LDL ingestion by $M_{1}$ and $M_{2}$ macrophages, $k_{L_{o x}}$ is ox-LDL saturation for production of MCP-1, $d_{L_{o x}}$ is degradation rate of ox-LDL. Equation (3) models the production of ox-LDL due to LDL oxidation by reaction with the radicals and a reduction of ox-LDL through ingestion by $M_{1}$ and $M_{2}$ macrophages [2].

Equation for free radicals:

$$
\frac{d r}{d t}=r_{0}+\lambda_{r} M_{1}-r\left(k_{L} L+k_{H} H\right)
$$

Here $r_{0}$ is influx of free radical into intima from the surrounding tissues, $\lambda_{r}$ is radicals production rate by macrophages $M_{1}$. Equation (4) models the evolution of radical concentration through a source term $r_{0}[2,3]$, production by macrophages $M_{1}[1]$ and reduction in concentration through oxidation reactions $[2,3]$.

Equations for monocytes:

$$
\begin{gathered}
\frac{d A_{1}}{d t}=\lambda_{P A_{1}} \frac{P}{k_{1 P}+k_{2 P} P} A_{1}^{0}-\lambda_{C_{1} A_{1}} \frac{C_{1}}{k_{C_{1}}+C_{1}+k_{4} C_{4}} A_{1}-d_{A_{1}} A_{1}, \\
\frac{d A_{2}}{d t}=\lambda_{P A_{2}} \frac{P}{k_{1 P}+k_{2 P} P} A_{2}^{0}-\lambda_{C_{2} A_{2}} \frac{C_{2}}{k_{C_{2}}+C_{2}} A_{2}-d_{A_{2}} A_{2} .
\end{gathered}
$$

Here $\lambda_{P A_{1}}$ and $\lambda_{P A_{2}}$ is rate of $A_{1}$ and $A_{2}$ monocytes penetration through the vessel wall, $A_{1}^{0}$ and $A_{2}^{0}$ is density of $A_{1}$ and $A_{2}$ monocytes in blood, $d_{A_{1}}$ and $d_{A_{2}}$ is death rate of $A_{1}$ and $A_{2}$ monocytes. Here $P:=C_{3}+L_{o x}$ corresponds to the activation of endothelial cells (pro-inflammatory action). Migration of smooth muscle cells depends on the activation of endothelial cells, and the activation of endothelial cells depends on $P$ [4], so the coefficient $k_{2 P}$ includes the activation of endothelial cells and the migration of smooth muscle cells. The first term is the influx of monocytes into the intima from the blood [1], the second term is the differentiation of monocytes into macrophages [5-7], and the third term corresponds to the death of monocytes.

Equations for macrophages:

$$
\frac{d M_{1}}{d t}=\lambda_{C_{1} A_{1}} \frac{C_{1}}{k_{C_{1}}+C_{1}+k_{4} C_{4}} A_{1}-\lambda_{L_{o x} M_{1}} \frac{L_{o x}}{k_{L_{o x}}+L_{o x}} M_{1}-d_{M_{1}} M_{1},
$$




$$
\frac{d M_{2}}{d t}=\lambda_{C_{2} A_{2}} \frac{C_{2}}{k_{A_{2}}+C_{2}} A_{2}-\lambda_{L_{o x} M_{2}} \frac{L_{o x}}{k_{L_{o x}}+L_{o x}} M_{2}-d_{M_{2}} M_{2} .
$$

Here first term is the differentiation of monocytes into macrophages [5-7], the second term describes transformation of macrophages into foam cells [2], and the third term corresponds to the death of macrophages.

Equations for T-helper cells:

$$
\begin{aligned}
& \frac{d T_{1}}{d t}=\lambda_{P T_{1}} \frac{P}{k_{1 P}+k_{2 P} P} T_{1}^{0}-d_{T_{1}} T_{1}, \\
& \frac{d T_{2}}{d t}=\lambda_{P T_{2}} \frac{P}{k_{1 P}+k_{2 P} P} T_{2}^{0}-d_{T_{2}} T_{2} .
\end{aligned}
$$

Here $\lambda_{P T_{1}}$ and $\lambda_{P T_{2}}$ is rate of $T_{1}$ and $T_{2}$ cells penetration through the vessel wall, $T_{1}^{0}$ and $T_{2}^{0}$ is density of $T_{1}$ and $T_{2}$ cells in blood, $d_{T_{1}}$ and $d_{T_{2}}$ is death rate of $T_{1}$ and $T_{2}$ cells. Equations (9) and (10) models the influx of T-cells from the blood into the intima (similar to monocytes) [1] and death of T-cells.

Equations for T-helper cytokines $C_{1}$ (IFN- $\gamma$, IL-1 $\beta$ ) and $C_{2}$ (IL-4, IL-13):

$$
\begin{aligned}
& \frac{d C_{1}}{d t}=\lambda_{C_{1}} T_{1}-k_{C_{1} A_{1}} C_{1} A_{1}-d_{C_{1}} C_{1}, \\
& \frac{d C_{2}}{d t}=\lambda_{C_{2}} T_{2}-k_{C_{2} A_{2}} C_{2} A_{2}-d_{C_{2}} C_{2} .
\end{aligned}
$$

Here T-cells produced $C_{1}$ and $C_{2}$ at a rate of $\lambda_{C_{1}}$ and $\lambda_{C_{2}}$, which promotes differentiation $A_{1}$ and $A_{2}$ monocytes into $M_{1}$ and $M_{2}$ macrophages at a rate of $k_{C_{1} A_{1}}$ and $k_{C_{2} A_{2}}$ and consumed (internalized) due to this interaction [6]. The third term corresponds to the degradation of cytokines at a rate of $d_{C_{1}}$ and $d_{C_{2}}$.

Equation for pro-inflammatory agents (IL-6, IL-12 and TNF- $\alpha$ ):

$$
\frac{d C_{3}}{d t}=\lambda_{C_{4} M_{1}} \frac{1}{k_{C_{4}}+C_{4}} M_{1}+\lambda_{C_{4} F} \frac{1}{k_{C_{4}}+C_{4}} F-d_{C_{3}} C_{3} .
$$

Here macrophages $M_{1}$ and foam cells $F$ produce pro-inflammatory cytokines $C_{3}$ at a rate of $\lambda_{C_{4} M_{1}}$ and $\lambda_{C_{4} F}$ activating endothelian cells [6]. It is promote cells penetration. The third term corresponds to the death of cytokines at a rate of $d_{C_{3}}$. Anti-inflammatory cytocines $C_{4}$ downregulate $C_{3}$ production.

Equation for anti-inflammatory agents (IL-10 and TGF- $\beta$ ):

$$
\frac{d C_{4}}{d t}=\lambda_{C_{4}} M_{2}-d_{C_{4}} C_{4}
$$

Here $M_{2}$ macrophages produce anti-inflammatory cytokines $C_{4}$ at a rate of $\lambda_{C_{4}}[5,6,10]$. It is prevent cells penetration. The second term corresponds to the degradation of cytokines.

Equation for foam cells:

$$
\frac{d F}{d t}=\lambda_{L_{o x} M_{1}} \frac{L_{o x}}{k_{L_{o x}}+L_{o x}} M_{1}+\lambda_{L_{o x} M_{2}} \frac{L_{o x}}{k_{L_{o x}}+L_{o x}} M_{2}-d_{F} F
$$

Here $M_{1}$ and $M_{2}$ macrophages that have ingested a large amount of ox-LDL become foam cells [2]. The third term corresponds to the death of foam cells. 


\section{Reduced model}

Let us consider the particular case where $H=A_{2}=M_{2}=T_{2}=C_{2}=C_{4}=0$, $r=R_{0}=$ const, $A_{1}=A_{1}^{0}=$ const. From equation (5), assuming a quasi-stationary approximation and $\lambda_{C_{1} A_{1}}=0$, we express $A_{1}$ through $P$ and substitute into equation (7). Furthermore, if $C_{1}$ is sufficiently large, the term $C_{1} /\left(k_{C_{1}}+C_{1}+k_{4} C_{4}\right)$ can be approximated by a constant. Thus, system (1)-(15) can be reduced to the following system of five equations:

$$
\begin{gathered}
\frac{d L}{d t}=\sigma_{L}\left(L_{0}-L\right)-k_{L} R_{0} L, \\
\frac{d L_{o x}}{d t}=k_{L} R_{0} L-\lambda_{1} \frac{L_{o x}}{k_{1}+L_{o x}} M_{1}-d_{1} L_{o x}, \\
\frac{d M_{1}}{d t}=\lambda_{2} \frac{C_{3}+L_{o x}}{k_{2}+C_{3}+L_{o x}}-\lambda_{1} \frac{L_{o x}}{k_{1}+L_{o x}} M_{1}-d_{2} M_{1}, \\
\frac{d C_{3}}{d t}=\lambda_{3} \frac{C_{3}}{k_{3}+C_{3}} M_{1}-d_{3} C_{3}, \\
\frac{d F}{d t}=\lambda_{1} \frac{L_{o x}}{k_{1}+L_{o x}} M_{1}-d_{4} F,
\end{gathered}
$$

where $\lambda_{1}=\lambda_{L_{o x} M_{1}}, \lambda_{2}=k_{4} \lambda_{C_{1} A_{1}}, \lambda_{3}=k_{5} \lambda_{C_{4} M_{1}}$ and $d_{1}=d_{L o x}, d_{2}=d_{M_{1}}, d_{3}=d_{C_{3}}, d_{4}=d_{F}$.

It is easy to see that system (16)-(20) for $\lambda_{1}=0$ reduces to the system of two equations

$$
\begin{gathered}
\frac{d M_{1}}{d t}=\lambda_{2} \frac{C_{3}+L_{o x}^{*}}{k_{2}+C_{3}+L_{o x}^{*}}-d_{2} M_{1}, \\
\frac{d C_{3}}{d t}=\lambda_{3} \frac{C_{3}}{k_{3}+C_{3}} M_{1}-d_{3} C_{3},
\end{gathered}
$$

where $\left(L^{*}, L_{o x}^{*}\right)$ is the equilibrium point of system (16)-(17) for $\lambda_{1}=0$. The reduced system (21)-(22) of two equations is similar to the model considered in [11]. Thus, for $\lambda_{1}=0$ we know stationary points and the stability.

Then we analyze the system (16)-(20) for $\lambda_{1}>0$. The analysis shows that for $\lambda_{1}>0$, similar to the case $\lambda_{1}=0$, three cases are possible. Case 1: only one equilibrium point $E_{0}$ which is stable. Here

$$
E_{0}=\left(L^{*}, L_{o x}^{*}, M_{1}^{*}, 0, F^{*}\right), \quad M_{1}^{*}=\frac{\lambda_{2}}{d_{2}} \frac{L_{o x}^{*}}{k_{2}+L_{o x}^{*}}, \quad F^{*}=\frac{\lambda_{1}}{d_{4}} \frac{L_{o x}^{*}}{k_{2}+L_{o x}^{*}}
$$

is the easily determined equilibrium point of system (16)-(20) for $C_{3}^{*}=0$. The existence of other (for $C_{3}>0$ ) equilibrium points is determined, in fact, by the system (17)-(19). Excluding $C_{3}$ from the corresponding system of three algebraic equations, we obtain a system of two equations with respect to $L_{o x}$ and $M_{1}$ :

$$
\begin{gathered}
k_{L} R_{0} L^{*}-\lambda_{1} \frac{L_{o x}}{k_{1}+L_{o x}} M_{1}-d_{1} L_{o x}=0, \\
\lambda_{2} \frac{L_{o x}+\frac{\lambda_{3}}{d_{3}} M_{1}-k_{3}}{L_{o x}+\frac{\lambda_{3}}{d_{3}} M_{1}-k_{3}+k_{2}}+d_{1} L_{o x}-k_{L} R_{0} L^{*}-d_{2} M_{1}=0 .
\end{gathered}
$$

Three cases of solving system (23)-(24) are presented in Figure 2 - 4. In case 1, system (23)-(24) has no positive solutions (Figure 1) and, therefore, system (16)-(20) has only one equilibrium point $E_{0}$, which is stable. In case 2, system (23)-(24) has only one positive 
solution (Figure 2) and system (16)-(20) there are two equilibrium points $-E_{0}$ is unstable and $E_{1}$ is stable (the monostable case). The projection $E_{1}^{\prime}$ of the point $E_{1}$ on the plane $\left(L_{o x}, M_{1}\right)$ is shown in Figure 3. In case 3, system (23)-(24) has two positive solution (Figure 3) and system (16)-(20) there are three equilibrium points $-E_{0}$ and $E_{2}$ are stable and $E_{1}$ is unstable (bistable case). The projections $E_{1}^{\prime}$ and $E_{2}^{\prime}$ of the points $E_{1}$ and $E_{2}$ on the plane $\left(L_{o x}, M_{1}\right)$ is shown in Figure 4. Red lines in Figure $2-4$ represent graphs of function $M_{1}\left(L_{o x}\right)$, and blue lines represent hyperboles in variables $\left(L_{o x}, M_{1}\right)$ defined by system (23)-(24).

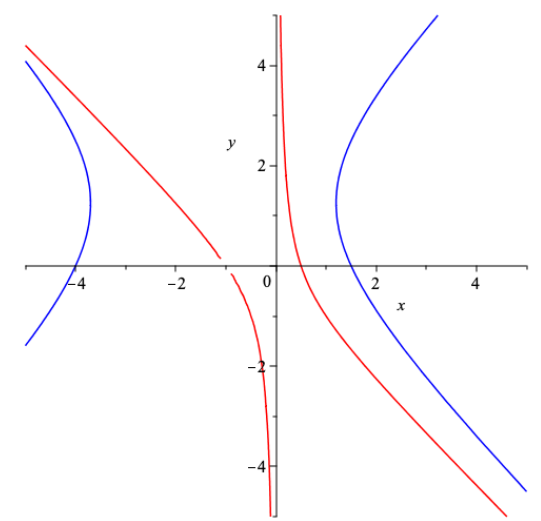

Figure 2. If $\lambda_{2} \lambda_{3}<k_{3} d_{2} d_{3}$ or $L_{0}$ small enough and $\lambda_{2} \lambda_{3}>k_{3} d_{2} d_{3}$, system (23)-(24) has no positive solutions.

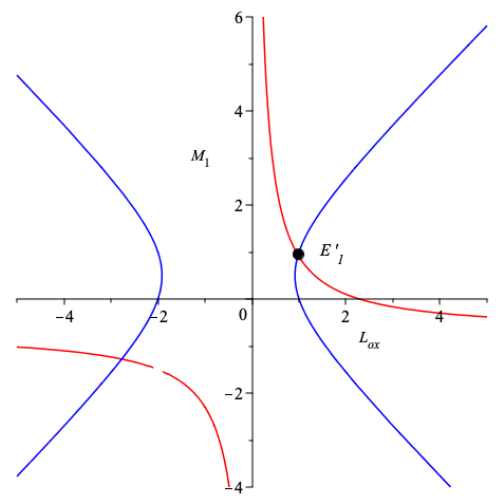

Figure 3. If $L_{0}$ large enough and $\lambda_{2} \lambda_{3}>$ $k_{3} d_{2} d_{3}$, system (23)-(24) has only one positive solution.

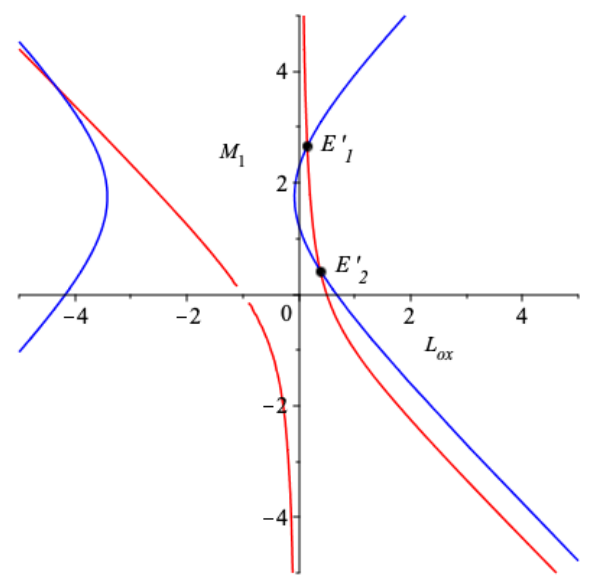

Figure 4. If $L_{0}$ is intermediate and $\lambda_{2} \lambda_{3}>k_{3} d_{2} d_{3}$, system (23)-(24) has two positive solutions.

Thus, the analysis of the model of five equations shows that the development of atherosclerosis depends on the concentration $L_{0}$ of low-density lipoproteins (bad cholesterol) in the blood. If this concentration is low, then there is only a disease-free equilibrium point, and atherosclerosis does not develop. For some intermediate values, there are three stationary 
points. Two of them, the disease-free point and the point corresponding to the disease development are stable. Each of them has its own basin of attraction. The system approaches to the second one if the initial perturbation of the disease-free point is sufficiently large. From the physiological point of view, it can be initiated by some additional factors such as inflammation or injury. Finally, for large concentrations of $L_{0}$, there are two stationary points. Thee disease-free equilibrium is unstable, and the disease will necessarily develop for any small perturbation.

\section{References}

[1] Orekhov A.N., Atherosclerosis (Palmarium Academic Publishing, Saarbrucken, Germany, 2013).

[2] Friedman A., Hao W., Bulletin of Mathematical Biology 77, Issue 5, 758-781 (2015).

[3] Hao W., Friedman A., PLoS ONE 9(3), 1-15 (2014).

[4] Harrington J.R., Stem Cells 18, 65-66 (2000).

[5] Gui T, Shimokado A, Sun Y, Akasaka T, Muragaki Y., Mediators Inflamm 2012, 1-14 (2012).

[6] Dipak P. Ramji, Thomas S. Davies, Cytokine and Growth Factor Reviews 26(6), 673-685 (2015).

[7] Gunter Almer, Daniela Frascione and et., Mol. Pharmaceutics 10, 175-186 (2013).

[8] Hansson G.K., Holm J., Jonasson L., Am. J Pathol. 135, 169-175 (1989).

[9] Barter P., European Heart Journal Supplements 7, 4-8 (2005).

[10] Xinbing Han, William A. Boisvert, The Role of IL-10 in Atherosclerosis (InTech, 2012).

[11] El Khatib N., Genieys S., Volpert V., Math. Model. Nat. Phenom. 2, No. 2, 126-141 (2007).

[12] Panfilov A.V., Dierckx H., Volpert V., Physica D 399, 1-34 (2019). 